\title{
THE DESIGN OF A MODULAR PROBE INCORPORATING THICK-FILM SENSORS FOR DETECTING PHASE INTERFACES IN OIL WELL PRIMARY SEPARATOR PLANT
}

 $n$ the extraction of hydrocarbons from the ground, the fluid pumped out of the well is not pure crude oil or natural gas, but a combination of oil, gas, and water, often containing entrained mud and particulate debris. This is usually a well-mixed multiphase flow, which often appears at the wellhead at high temperature and pressure.

It is necessary to separate out the phases as soon as possible after extraction. This is done in a vessel called a primary separator, which consists of a pack of parallel plates to promote separation and one or more weirs as shown schematically in Fig. 1. Although conceptually simple, these plate separators are difficult to operate because the interface levels cannot be seen. The high pressure and dirty environment prevent the use of a sight glass, and the high temperature and pressure make the use of conventional measurement or imaging instruments impractical.

This paper presents the development of a prototype sensor system for locating the phase interfaces in the harsh environment of a primary separator vessel. The specification adopted was:

- that it should comprise a portable device suitable for installing in a separator vessel through a standard "6inch flange" (a common size of standpipe penetration with an unimpeded bore diameter of about $125 \mathrm{~mm}$ found on separators and other pressure vessels) and

- that it be capable of working for extended periods in mixed-phase water/hydrocarbon environments at temperatures up to $150^{\circ} \mathrm{C}$ and pressures up to $15 \mathrm{MPa}(150 \mathrm{bar})$.

The first point means that the probe has to be of small cross section, no more than 125-mm diameter, and of variable length up to $3 \mathrm{~m}$ (a common diameter for separator vessels) or even more. This indicates the need for a modular design and ensures that the technology would be suitable for use in primary separators in most oil and gas fields. In terms of Fig. 1, a suitable location for a probe would be suspended vertically from a 6 inch flange inserted to the left of the standpipe marked "gas out" to hang between the end of the parallel-plate interceptor and the weir. In this position, it would enable the operator to ensure that the water was safely below the level of the weir and that the oil/water emulsion and oil/gas foam did not approach the "water out" and gas out standpipes, respectively.

The high-temperature and pressure requirement also make the technology suitable for most oil and gas fields worldwide

\footnotetext{
J.M. Hale is the reader in structural dynamics at the University of Newcastle upon Tyne, UK. N.M. White is the professor of thick-film materials at the University of Southampton, UK. A.J. Jaworski is a senior lecturer, G. Meng is a research student, and T. Dyakowski is the professor of chemical engineering at the University of Manchester, UK.
}

and also means that it could be adapted for use in a broad range of other chemical engineering applications and could potentially be used downhole in oil and gas wells.

\section{INTERFACE DETECTION: PROBLEMS AND PROPOSED SOLUTIONS}

The first problem was to identify a suitable measurement technique. The "interfaces" between phases are in practice not interfaces at all but mixed-phase regions: emulsion between the water and oil, and foams between the oil and gas. This makes detection quite difficult, so much so that experience indicated that it could not be done with a single measurement modality. ${ }^{1,2}$ Instead, it was proposed to combine electrical impedance and ultrasonic measurement techniques.

The second problem was the aggressive operating environment: a mixture of hydrocarbons and water, often strongly acidic, which may be at high temperature and pressure.

The hot acidic water would attack most materials, with the active faces of instruments being particularly vulnerable. This problem could be alleviated relatively easily by the use of stainless steel for most components. However, the impedance measurement instruments required electrodes in contact with the environment and insulated from any stainless steel components. It was proposed to use thick-film technology, using noble-metal-based conductive films deposited on alumina substrates.

An operating temperature specification of $150^{\circ} \mathrm{C}$ was adopted as typical of hot wells. This precludes the siting of any active electronics, and most polymers, within the separator vessel. It was proposed to use capacitance measurement for the electrical modality. It had been established previously ${ }^{3}$ that this can be done via up to $3 \mathrm{~m}$ of cable, provided it is carefully screened, and so can have the instrumentation located outside the separator vessel. The specified temperature made it possible to use polytetrafluoroethylene (PTFE) $\left(\right.$ Teflon $^{\circledR}$ ) insulated cable and Viton ${ }^{\circledR}$ (a high-temperature rubber) for O-ring seals.

The high pressure makes it difficult to design instruments that seal effectively and so maintain the integrity of the separator, a certified pressure vessel. Sealing with high-temperature O-ring seals was understood at the outset of the project, but the problem of making electrical penetrations for signal wires through the probe body to the instruments had no solution at the start of the project.

It was proposed to design a measurement probe that would be up to $3 \mathrm{~m}$ long and no more that 120-mm diameter 


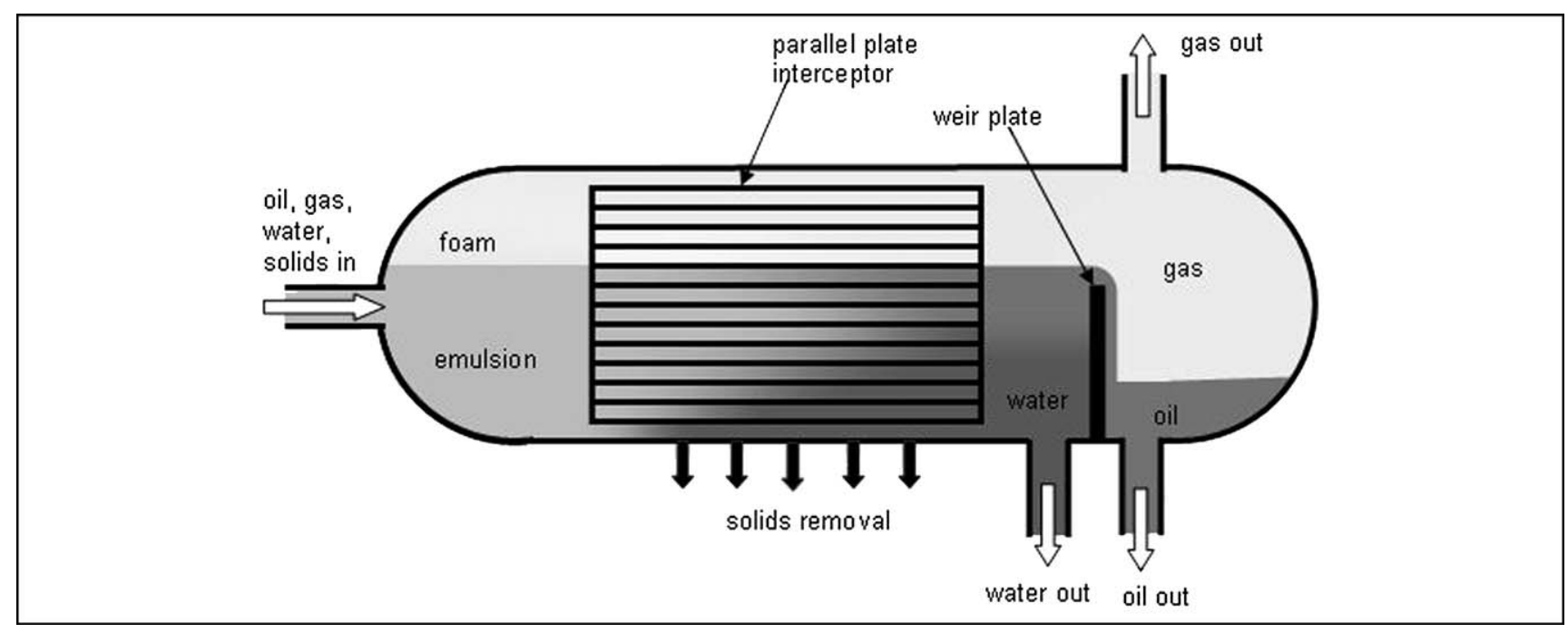

Fig. I: Schematic representation of a parallel-plate primary separator. The parallel plates help the phases to separate as they flow from left to right. There are no clear-cut boundaries between the phases; they blend into one another as foams and emulsions

to pass through a standard 6-inch flange and span the diameter of a separator vessel. The probe would contain multiple capacitance and ultrasonic transducer pairs facing one another across a narrow gap through which the process fluid could pass freely. In this way, the probe would measure the properties of the fluid at multiple locations across the vessel diameter without significantly affecting the flow.

\section{SENSOR MATERIALS DEVELOPMENT}

The first problem to be solved was to find suitable materials for the sensors. Although it was recognized that it might be possible to protect the sensors with a suitable barrier layer, it is desirable that the electrodes be in direct contact with the process fluids to maximize sensitivity and eliminate the need to compensate for the effects of the coating.

In previous work at lower temperatures, the Manchester authors used multilayer printed circuit technology to fabricate capacitance sensors. ${ }^{3}$ This used epoxy as the base material, which has been shown by the Newcastle group to be unsuitable for use at $150^{\circ} \mathrm{C}$. ${ }^{4}$ However, previous work at Southampton ${ }^{5}$ had developed the techniques for depositing conductive thick-films onto alumina tiles, and this technology was adopted.

Two candidate conductive thick-film materials were identified: silver-palladium and gold, both in the form of fine flakes dispersed in a glass matrix. To test their suitability, specimens were made up on 0.635 -mm-thick alumina tiles, each with a $10-\times 10-\mathrm{mm}$ conductive film on one side. These were subjected to emersion in water at $150^{\circ} \mathrm{C}$ and $15 \mathrm{MPa}(150 \mathrm{bar})$ for a period of 7 days, following which no change in the resistance of either film type could be detected using a handheld multimeter. A visual inspection showed some discoloration of the alumina around the silver-palladium, which was believed to be due to leeching of the silver component. There was no such effect in the gold film.
Although the discoloration around the silver-palladium films caused no measurable effect, it was decided to use the gold thick-film material for the sensor electrodes because the extra cost is moderate for the small quantities used.

Piezoelectric thick-film pastes have been developed previously at Southampton University ${ }^{5}$ and it was expected that the same formulation could be used again, particularly because the piezoelectric film would be isolated from the environment by the conductive electrode film. The piezoelectric material used previously was lead zirconate titanate (PZT), and no other material was seriously considered here because no other material approaches the piezoelectric sensitivity of PZT. The only question was the variety of PZT to be used. PZT-5H is the highest sensitivity form, but it has a Curie temperature quoted as $195^{\circ} \mathrm{C}$. $^{6}$ This is the temperature above which ferroelectric materials such as PZT spontaneously lose their piezoelectric properties and is analogous to the Curie temperature in ferromagnetic materials where spontaneous demagnetization occurs. Although this appears to be comfortably above the working temperature of $150^{\circ} \mathrm{C}$, it was known that depoling of piezoelectric materials occurs progressively and it was felt necessary to test its stability.

Trial piezoelectric transducers were fabricated onto 0.635-mmthick alumina tiles as shown in Fig. 2. These were found to work effectively as ultrasonic transmitters as well as receivers, and this suggested an effective means of testing their degradation with time. A tile was mounted in a lens carrier on an optical bench facing an instrument microphone connected to an oscilloscope via a calibrated amplifier. It was driven at about $10 \mathrm{kHz}$ with a constant amplitude of $20 \mathrm{~V} \mathrm{p}$-p, varying the frequency until the microphone signal registered a maximum, indicating a resonance of the tile. The microphone was then moved axially, which again varied the microphone signal due to standing waves set up in the sound field in front of the tile. When a maximum was achieved, the resonant frequency and received amplitude were noted. After the tiles were subjected to the test environment (water at $150^{\circ} \mathrm{C}$ and $15 \mathrm{MPa}$ ) for a week, they were retested in the same way for comparison. 


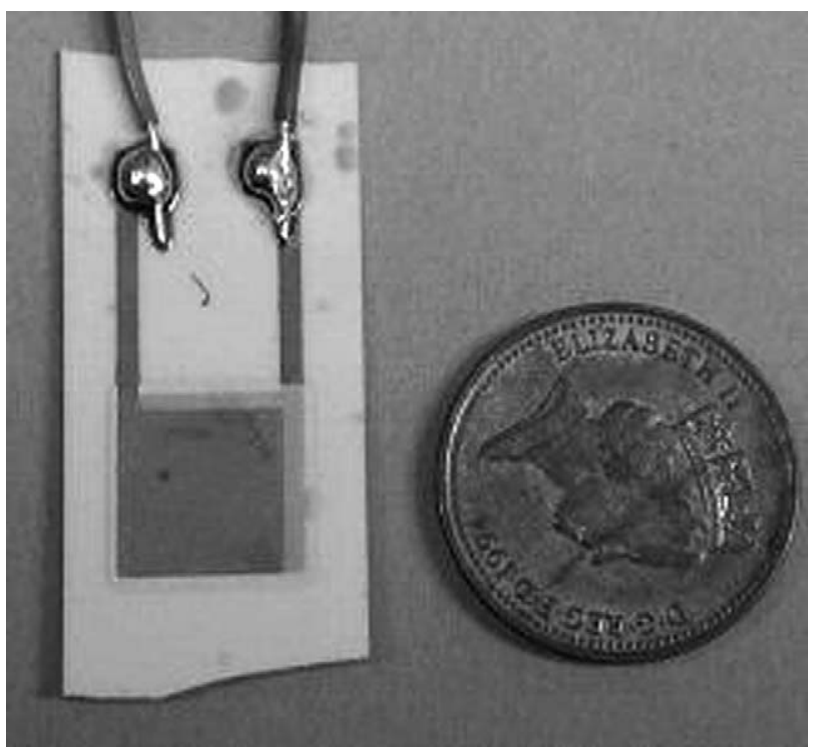

Fig. 2: Trial thick-film piezoelectric transducer. Thick-film sensors were deposited onto alumina tiles by screen printing and high-temperature curing. They comprise three layers: two conductive electrode layers sandwiching a piezoelectric layer

It was found that transducers made with PZT-5H thick film lost their piezoelectric properties after 7 days of emersion in the test environment. Fortunately PZT-5A, another form of PZT with lower sensitivity, has a quoted curie temperature of $350^{\circ} \mathrm{C}{ }^{6}$ Specimen transducers were made up in the same way using a thick-film paste containing PZT-5A and found to survive emersion in the test environment satisfactorily. Five specimens were tested in this way and a mean drop in sensitivity of $2.4 \mathrm{~dB}$ was observed. This is similar to the loss that always occurs in this type of transducer over its first few months and appears to be simply accelerated aging caused by the high temperature. It did not prevent the transducers from performing satisfactorily.

\section{SENSOR GEOMETRY DEVELOPMENT}

Having decided on the sensor materials, piezoelectric transducer tiles of various sizes were made up for functional testing. These were mounted on a fork structure in pairs facing one another and immersed in a tank of liquid at moderate temperatures and ambient pressure to form the test environment. By this means, the transducers could be used both as a "pitch/catch" ultrasonic pair and as a capacitance transducer using only the two outer electrodes in contact with the liquid.

As expected, it was found that large electrodes gave a high sensitivity to the capacitance modality of the sensor but were detrimental to the accuracy with which the ultrasonic pair could be used to measure time of flight. With a gap of 22 $\mathrm{mm}$ (the minimum judged necessary to ensure good flow through an instrument in a separator vessel), capacitor electrodes of $35 \times 35 \mathrm{~mm}$ were found to give satisfactory results, but the preferred size for the ultrasonic transducers was only $10 \times 10 \mathrm{~mm}$.
It was still considered desirable to use the sensors for both measurement modalities. This was achieved using dissimilarsized electrodes as shown in Figs 3 and 4. The smaller inner electrode effectively defines the size of the ultrasonic transducer because it controls the extent of the electric field in the piezoelectric layer sandwiched between them, but when used in the capacitance and conductance modes, the inner electrodes have no effect and the outer electrodes define the size of the electrical sensor. Asymmetrical sensor tiles of this type were made up and tested in water, saline solution, and vegetable oil and found to work as expected.

Using these sensor tiles, a series of tests was carried out to establish the viability of identifying oil, water, and gas phases and the effects of temperature and the concentrations of emulsions and foams. This is described fully elsewhere. ${ }^{7}$

\section{PROBE DEVELOPMENT}

The probe was conceived as an inverted pressure vessel, with the inside of the probe body communicating with the atmosphere and the outside exposed to the high pressure of the separator vessel. This arrangement has the advantage that most of the wiring is out of contact with the process fluids and could use conventional PTFE-insulated screened cable of the type commonly used for piezoelectric sensors such as accelerometers.

There were three problems to be overcome:

- to make electrical penetrations through the probe body for the connections to the electrodes on the sensor tiles,

- to devise a geometry that would provide an unimpeded 22-mm gap between sensor tiles and sufficiently large crush-proof channels to accommodate the wiring, all fitting within the 120 -mm-diameter envelope required for access through a 6 -inch flange, and

- to make the device modular so that a 3-m-long probe could be constructed on site from components that might be conveniently transported and handled.

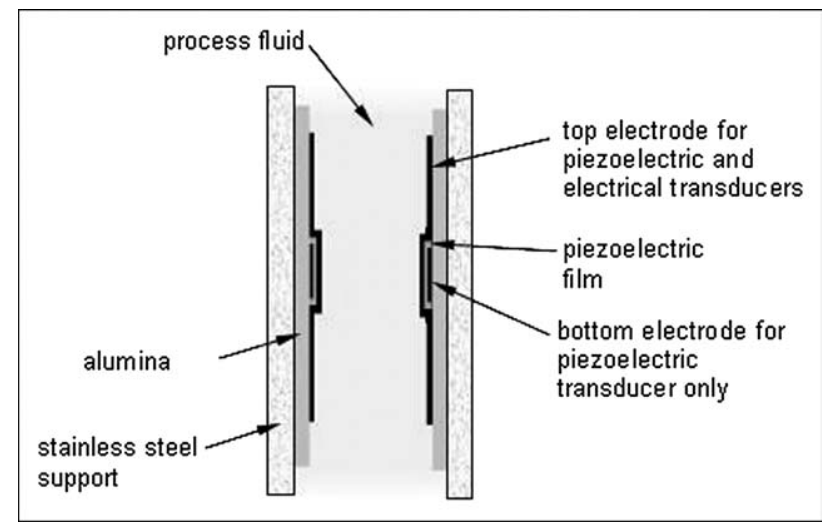

Fig. 3: Dual-modality transducer. Each transducer consists of two electrodes sandwiching a piezoelectric layer to form a piezoelectric transducer suitable for sending and receiving ultrasound. The outer electrodes in contact with the process fluid are also used as conductance and capacitance sensors to measure electrical properties 


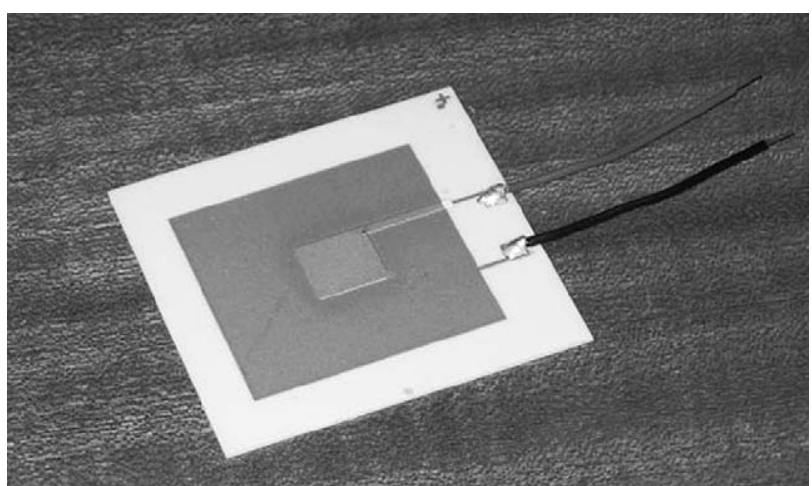

Fig. 4: Transducer tile with dissimilar-sized electrodes. A small electrode is deposited on the alumina tile and covered with a piezoelectric layer, which is in turn covered by a larger electrode layer. The electrodes are isolated by the piezoelectric layer, which is a dielectric insulator. The dimensions of the piezoelectric transducer are defined by the smaller electrode

\section{ELECTRICAL PENETRATIONS}

The problem was to find a method of transmitting sensor signals through the wall of the probe without compromising its integrity as a pressure vessel and maintaining adequate screening of the very sensitive capacitance signals.

Obtaining adequate screening was straightforward and required only that each penetration pin went through an individual hole so that the probe body became its screen. It was necessary only to find a way to seal the pins without making electrical contact with the hole.

Early trials using high-temperature epoxy adhesive were not successful. From previous work, ${ }^{4}$ the authors were well aware of the plasticizing effect of water absorption into epoxy resin, which reduces its glass transition temperature $\left(T_{\mathrm{G}}\right)$. This absorption occurs very rapidly at high temperatures but is limited to a surface layer. It was anticipated that the combination of the deep hole and relatively thin epoxy layer would maintain the pressure tight seal even though the outer layers of the seal were working above $T_{\mathrm{G}}$. It was found that a suitable aerospace grade of epoxy (Vantico AV119, Duxford, UK) did seal effectively, but it was not found possible to isolate the penetration pin reliably with a thin epoxy film, even when using taper pins in taper-reamed holes.

The problem was eventually solved by a commercial supplier, Wesley Coe Ltd of Cambridge, UK. They produced stainless steel bodies with through holes in which were mounted nickelplated copper pins sealed with glass as shown in Fig. 5. A large radial gap (approximately $1 \mathrm{~mm}$ ) around the pins ensures reliable insulation. The penetrator body was designed for mounting in a vessel by electron beam welding. However, it was found that it could be retained and sealed effectively by means of the high-temperature epoxy AV119 in a stepped seat, arranged such that the high external pressure forces the penetrator body onto its seat. After soldering wires to the pins, the space was potted with a high-temperature grade of silicone elastomer (Dow Corning Sylgard 182, Midland, MI) to ensure that the pins could not bend and make contact with the body.

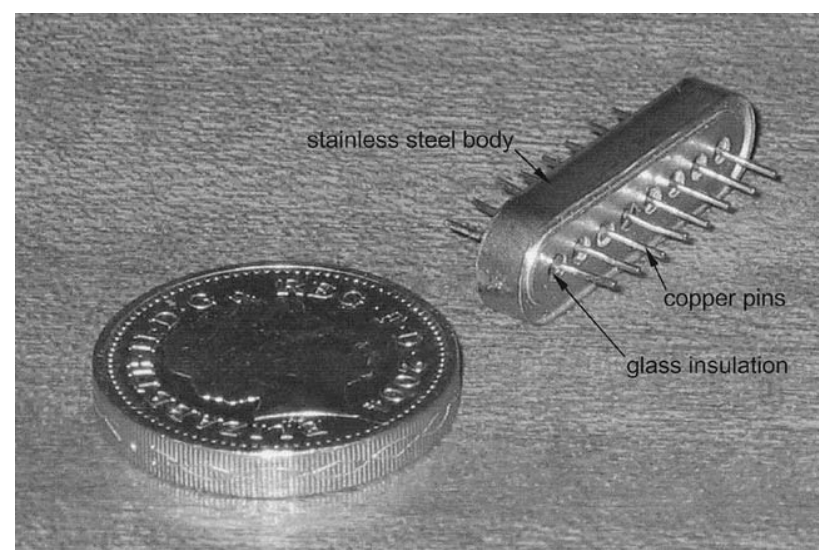

Fig. 5: Electrical penetrator

\section{MODULAR PROBE BODY}

The high external pressure acting on the probe body indicated a solid structure to prevent crushing. Channels for internal wiring could be drilled without introducing large stresses. The problems were obtaining access to the inside of the penetrator pins to solder on the wires, sealing between modules, and drilling long internal bores in stainless steel to connect up multiple sensors.

The solution finally arrived at is shown in Figs. 6-8. The probe modules are made short, each module housing a single electrical/ultrasonic sensor pair, which eliminates the need for long-hole drilling. The probe bodies are machined from solid billets of 316 stainless steel and located at a fixed distance apart by four pillars. This gives adequate strength and stiffness to form a stable platform for the sensors and to withstand the external pressure.

All sealing is done using Viton high-temperature O-rings configured as radial seals. Face seals, which are difficult to set up

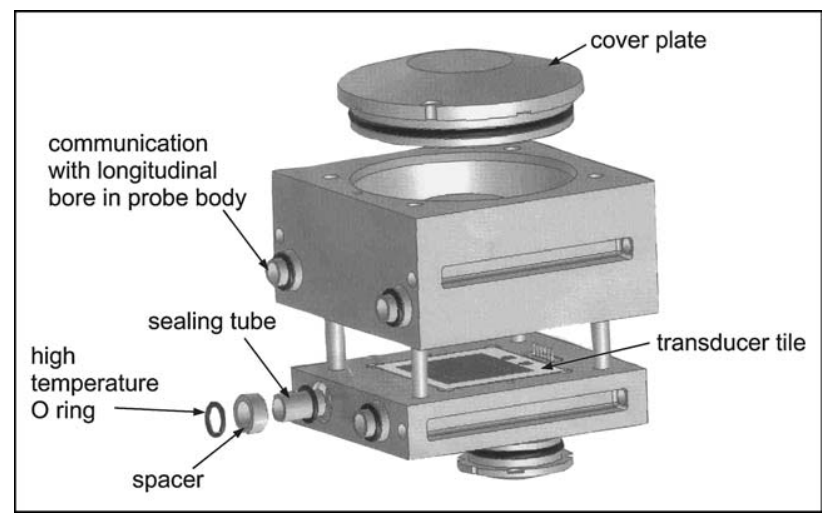

Fig. 6: Modular probe. The probe is made up of a number of these modules, connected longitudinally. Signal wires pass through the probe bodies, and hence to the outside of the separator vessel, through longitudinal bores. Interconnection between modules is by means of short tubes with O-rings. Sealing is achieved between the outer diameter of the tubes and the bore of a circular recess in the probe body, eliminating the need for face seals 


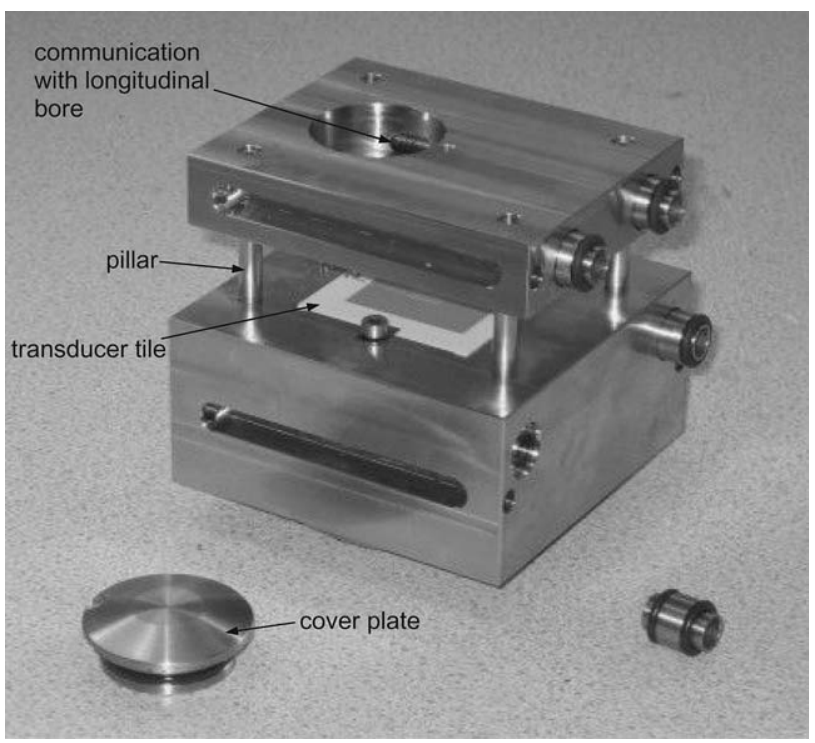

Fig. 7: Photograph of a single probe module. The transducer tiles are held at a constant distance apart by mounting on rigid bodies, held together by four pillars. Access to the penetrator for soldering signal wires is by means of a circular hole communicating with the longitudinal bores. These access holes are filled in use with an O-ring sealed cover, stepped such that it is held in place by the external pressure

reliably, have been eliminated as described below. The covers for the penetration pin access chambers are cylindrical plates held in place against the pressure by a lip and sealed with a conventional piston-type $\mathrm{O}$-ring groove.

The seals between modules are shown in Fig. 6. They are required to allow the transducer leads to be taken to the end of the probe assembly and hence out of the separator vessel. The obvious way to achieve this would be to use face seals such as gaskets, but these would be difficult to make reliably in a long structure of this type where significant bending loads might occur, tending to open up the faces. The arrangement shown allows the pressure to penetrate the faces to the inner tubes, which are sealed in both of two adjoining modules by two O-rings. The seal is made between the inner tube and the outer bore via the O-ring and is purely radial, very much like a piston seal. The outer tube is only a spacer to ensure that the O-rings seat in both modules and does not form part of the seal.

The arrangement has three advantages.

- It is not subject to leaking if the axial load on the interface between modules should be uneven.

- The components, stainless steel tubes and Viton O-rings, are small and low cost and the small-diameter tubes are stable against external pressure.

- A long probe made up of many modules will be stable against buckling because the effective axial load is relatively small. This is because the pressure penetrates through most of the intermodular gap and the axial load is only the product of the external pressure and the area of the four inner tubes.

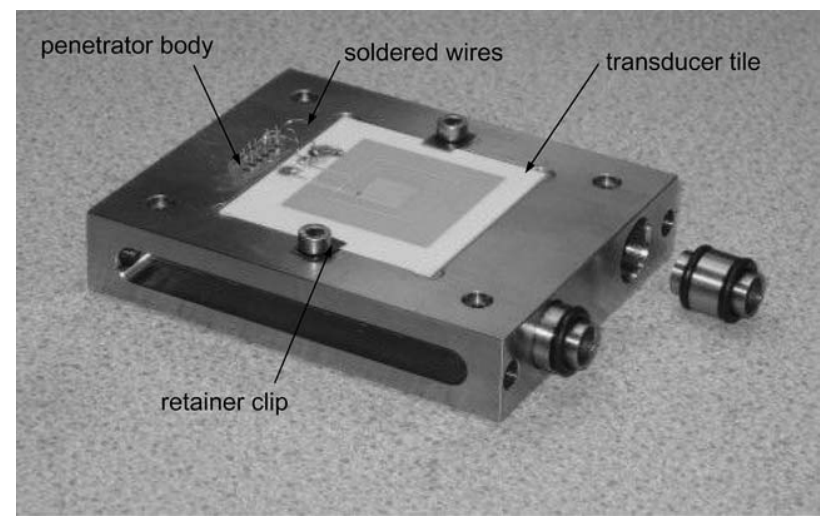

Fig. 8: Photograph of one half of a probe module. The transducer tiles are retained by clips to allow for differential thermal expansion. Electrical connections between the penetrator bodies and transducer electrodes are by fine soldered wires, subsequently potted for environmental and abrasion protection

The only potential difficulty with this design is that it requires accurate machining to ensure that the O-ring bores line up between pairs of assembled probe modules. However, this is well within the capabilities of computer numerical control (CNC) machining and no difficulties were experienced.

The modules are held together by tie bars running the length of the probe. In fact the external pressure would be more than sufficient to hold the probe together in operation, and the tie bars are provided only to maintain integrity in the event of an accident and to facilitate handling during installation.

\section{TESTING THE PROTOTYPE PROBE}

As noted above, the sensor tiles were tested at an early stage in this work, and the results have been published previously. ${ }^{7}$ Subsequently, a number of probe modules were made up for functional testing. Some preliminary results are given here.

Figure 9 shows a single probe segment with stainless steel casings, immersed in a glass test vessel filled with crude oil/process water mixture. The inset shows the "tiles-alone" arrangement that would be placed in the same glass vessel with a smaller quantity of mixture. In both cases, the homogenizer was used to provide a uniform oil-water emulsion.

The probe was set up as described above so that two sensor tiles face one another across a gap of $22 \mathrm{~mm}$, immersed in a tank containing the test fluid. The signal wires were led out of the tank for connection to an ultrasonic flaw detector set (Panametrics EPOCH II Model 2100, Waltham, MA) and an inductancel capacitance resistance (LCR) meter (Hioki 3532-50 LCR HiTester, Nagano, Japan) for making very accurate measurements of electrical resistance and capacitance, as described fully in Meng et al. ${ }^{7}$

A set of experiments was undertaken to measure the sonic velocity (from time of flight across the known gap), capacitance, and conductance (again for electrodes of known dimensions 


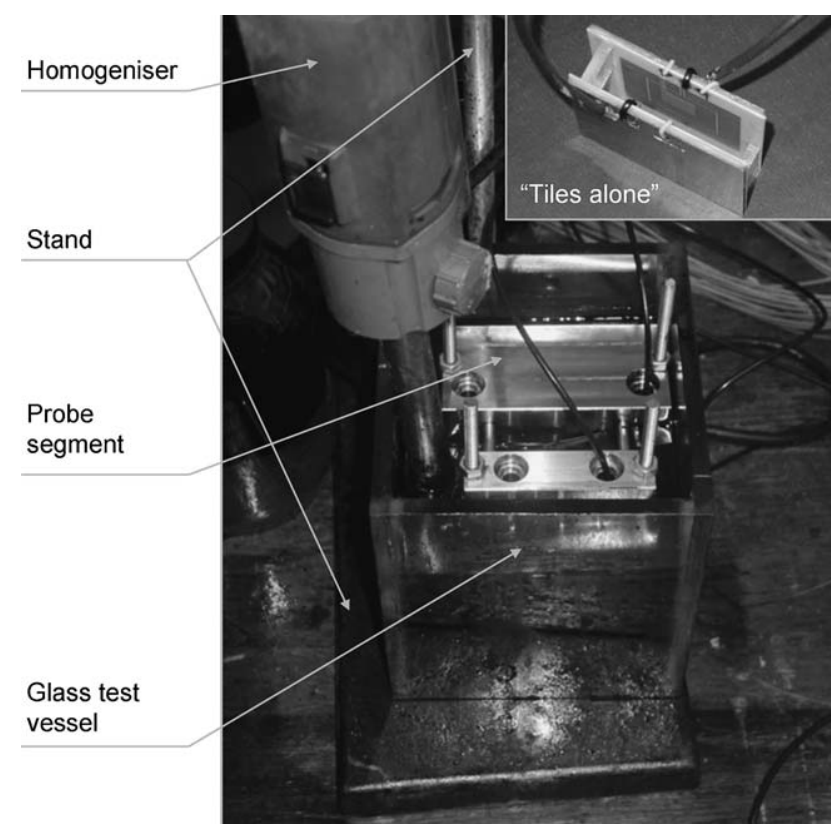

Fig. 9: Photograph of environmental testing arrangement. A probe segment is immersed in the test environment above the level of the sensor tiles. Inset is the arrangement used to test the tiles alone before the probe bodies were made

across a known gap). For this initial set of tests, the temperature was held constant at $25^{\circ} \mathrm{C}$ and the test fluid was an $\mathrm{oil} /$ water emulsion of varying composition.

The test fluids were of standard type supplied by the National Engineering Laboratory. The oil component was a mixture of Forties and Beryl crude oils, topped to remove light ends and increase flashpoint to $75^{\circ} \mathrm{C}$, with kerosene added to restore the original viscosity. The water was simulated process water, a solution of magnesium sulfate heptahydrate $\left(\mathrm{MgSO}_{4} \cdot 7 \mathrm{H}_{2} \mathrm{O}\right.$, commonly known as epsom salts) of concentration $75 \mathrm{~kg} / \mathrm{m}^{3}$. The emulsion was prepared according to the methods described fully in Meng et al. ${ }^{7}$

To validate the probe, a representative sample of measurements were made using unmounted sensor tiles (as described previously in Meng et al. ${ }^{7}$ but using the crude oil/simulated process water in place of the vegetable oil/saline used in the preliminary trials) and compared with the same measurements using the tiles mounted in a probe. The results are given in Figs. 10, 11, and 12, showing variation of sonic velocity, capacitance, and conductance as a function of oil concentration, respectively.

Figure 10 shows that the probe body has no significant effect on sonic velocity. The small differences are equivalent to discrepancies of no more than $50 \mathrm{~ns}$, which is within the resolution of the measurement system. The relationship between sonic velocity and oil concentration is not quite linear, but it is a clear one-to-one relationship that could easily be used to infer oil concentration.

The capacitance measurements (Fig. 11) taken with the unmounted sensor tiles show a well-behaved monotonic

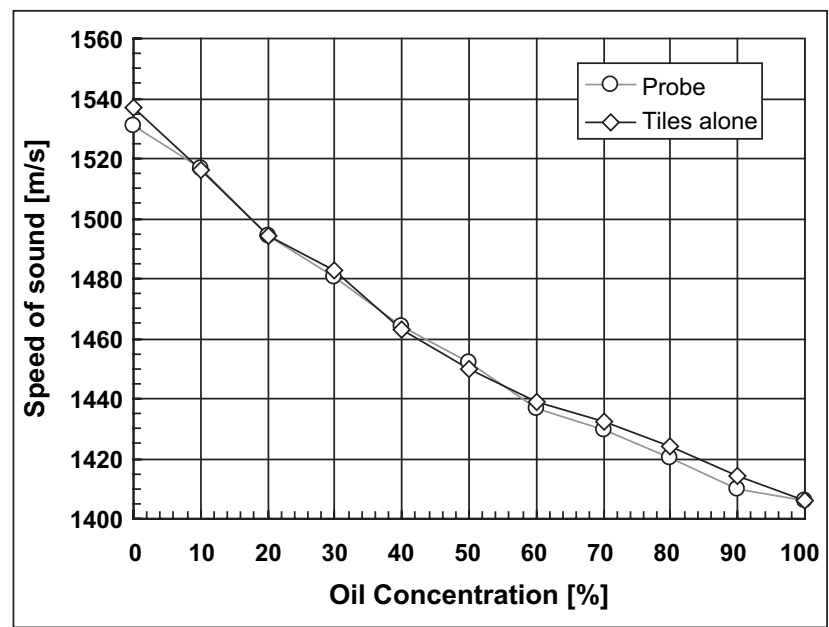

Fig. 10: Sonic velocity of oil/water emulsions at $25^{\circ} \mathrm{C}$. The velocities measured by the probe do not differ significantly from those measured using unmounted transducer tiles, indicating that the probe body has no effect on this measurement

relationship between the oil concentration and the capacitance values, though with some scatter about an idealized smooth line at low oil concentrations. This is due to the difficulty in measuring the dielectric properties of water-continuous oil-water mixtures accurately because of their relatively high conductivity. The anomalous value obtained at 50\% concentration is probably due to the slightly erratic properties of the mixture where the continuity cannot be predicted.

Clearly, mounting the sensors within steel probe body changes the capacitance results markedly. At oil concentration above $30 \%$, there is a consistent capacitance reduction of about $30 \%$ (apart form the anomaly at $50 \%$ oil concentration mentioned above). It is suggested that this is due to the

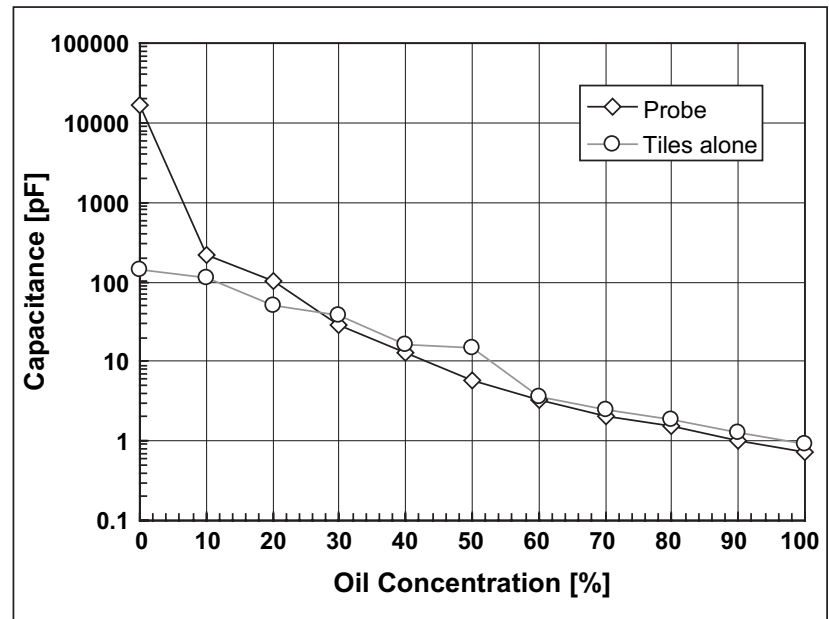

Fig. II: Capacitance of oil/water emulsions at $25^{\circ} \mathrm{C}$. Above $30 \%$ oil concentration, the probe body causes a significant but uniform reduction in capacitance, but below $30 \%$ concentration, it causes a large increase in capacitance. This is due to the effect of the conductive pillars separating the probe bodies and the high permittivity of the water phase 


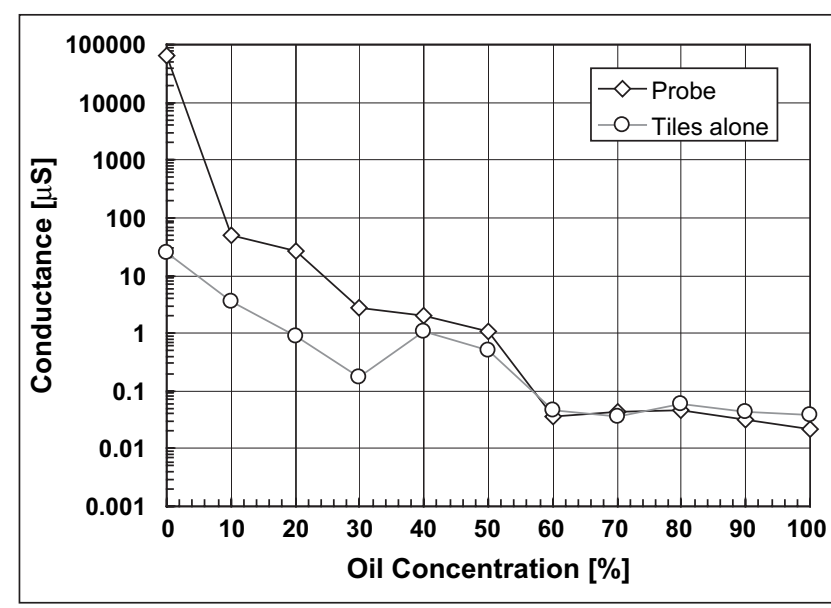

Fig. 12: Conductance of oil/water emulsions at $25^{\circ} \mathrm{C}$. At high oil concentrations, the probe body has little effect on measured conductance. At oil concentrations below $60 \%$, the lowresistance pillars separating the probe bodies become significant

presence of the steel structure of the probe body distorting the electric field and hence increasing the apparent distance between the electrodes forming the capacitor plates. However, at low oil concentrations, the capacitance is greatly increased, which may be due to the high permittivity water allowing the field to migrate into the pillars separating the halves of the probe body which, being conductors, will be at almost constant potential. This will have the effect of reducing the apparent gap between the electrodes and hence greatly increasing the capacitance.

In any event, the practical conclusion is that for a fixed configuration of casings and sensors, there is a clear "calibration" line that can be used to infer the media composition from the measured values of capacitance.

The conductance measurements in Fig. 12 show good agreement at high oil concentrations above $60 \%$, but below this value, the probe indicates far higher conductance, and hence apparent conductivity, of the fluid mixtures. For the unsupported sensor tiles, the electrical path is directly from one tile (electrode) to the other. The process water will form a moderately good electrolyte and so conduct well while there is a connective path through it, but at high oil concentrations, the water will form drops within the oil matrix, so its electrolytic properties will be ineffective. With the tiles mounted in the probe, the electrical path between the electrodes will be through the pillars, that is, two high resistances (the track through the fluid across the surfaces of the tiles) in series with the low-resistance pillars. This will make the resistance relatively low (high conductance) at low oil concentrations as the path through the electrolyte is short but will have no effect at high oil concentrations when the fluid is effectively insulating along any path.
These results make two important points for practical measurement of water/oil emulsion concentration and hence detection of phase interfaces in separator plant. First, the techniques are not restricted to the clean vegetable oils and saline water used in the early trials ${ }^{7}$ but work as well with crude oil and process water of the type encountered in real oil extraction. Second, the measurements are not disrupted by the presence of the heavy steel probe body in close proximity to the sensor tiles.

\section{CONCLUSIONS}

A robust prototype multimodality (capacitance, conductance, and ultrasonic) measurement probe has been built and tested. Its principal features are:

- high-strength construction using stable materials suitable for extreme operating temperatures and pressures up to $150^{\circ} \mathrm{C}$ and 150 bar,

- modular construction to enable long probes to be constructed on site,

- small cross-section to present minimal flow restriction within the vessel and enable deployment in pressure vessels through standard 6-inch flanges.

Measurements made using the probe have been shown to be similar to those made with the sensors in isolation. The probe is able to measure oil concentration in oil/water and water/oil emulsions.

\section{ACKNOWLEDGEMENT}

The authors acknowledge the support of the Engineering and Physical Sciences Research Council whose grant made this work possible.

\section{References}

1. Beck, M.S., Byars, M., Dyakowski, T., Waterfall, R., He, R., Wang, S.J., and Yang W.Q., "Principles and Industrial Applications of Electrical Capacitance Tomography," Measurement and Control 30(7):197-200 (1997).

2. Hale, J.M., "Ultrasonic Density Measurement for Process Control," Ultrasonics 26:356-357 (1988).

3. Jaworski, A.J., Dyakowski, T., and Davies, G.A., "A Capacitance Probe for Interface Detection in Oil and Gas Extraction Plant," Measurement Science \& Technology 10:L15-L20 (1999).

4. Hale, J.M., Gibson, A.G., and Speake, S.D., "Biaxial Failure Envelope and Creep Testing of Fibre Reinforced Plastic Pipes in High Temperature Aqueous Environments," Journal of Composite Materials 36(3):257-270 (2002).

5. Maas, R., Koch, M., Harris, N.R., White, N.M., and Evans, A.G.R., "Thick-film Printing of PZT onto Silicon," Materials Letters 31:109-112 (1997).

6. Piezoelectric Ceramics Data Book for Designers, Morgan Electroceramics Ltd, Thornhill, Southampton, UK.

7. Meng, G., Jaworski, A.J., Dyakowski, T., Hale, J.M., and White, N.M., "Design and Testing of a Thick-film Dual-modality Sensor for Composition Measurements in Heterogeneous Mixtures," Measurement Science \& Technology 16:1-13 (2005). 